\title{
Laina: Dynamic Data Physicalization for Slow Exercising Feedback
}

\author{
Daphne Menheere \\ Eindhoven University of Technology, \\ Department of Industrial Design, the \\ Netherlands
}

\author{
Evianne van Hartingsveldt \\ Eindhoven University of Technology, \\ Department of Industrial Design, the \\ Netherlands
}

\author{
Mads Birkebæk \\ Aarhus University, Computer Science, \\ Denmark
}

\begin{abstract}
Steven Vos
Eindhoven University of Technology,

Department of Industrial Design, the

Netherlands; Fontys University of

Applied Sciences Eindhoven, the

Netherlands
\end{abstract}

\author{
Carine Lallemand \\ Eindhoven University of Technology, \\ Department of Industrial Design, the \\ Netherlands; University of \\ Luxembourg, Esch-sur-Alzette, \\ Luxembourg
}

\begin{abstract}
The increased popularity of recreational sports, like running, led to the development of numerous technologies supporting people in their training. However, in their current form and interaction, these take a rather standardized approach focusing on quantified data tracking displayed through screens or audio. In this paper, we explore how dynamic data physicalization through a shapechanging interface can open the design space of exercise feedback. Relying on an expert study on the aesthetics of interaction $(\mathrm{N}=23)$, we designed Laina, a shape-changing art piece presenting physicalized running data through a slow feedback mechanism. We deployed Laina at 3 participant's home, during a series of 3-weeks field studies. Results show that Laina allows for deep reflection, anticipation and exploration of running behavior. The aim of our paper is to provide insights on the use of slow feedback mechanisms for exercise-related products, through the design of a dynamic data physicalization artefact.
\end{abstract}

\section{CCS CONCEPTS}

- Human-centered computing $\rightarrow$ Human computer interaction (HCI); Interaction devices.

\section{KEYWORDS}

Data physicalization, shape-changing interface, exercise feedback, recreational runners, slow feedback

\section{ACM Reference Format:}

Daphne Menheere, Evianne van Hartingsveldt, Mads Birkebæk, Steven Vos, and Carine Lallemand. 2021. Laina: Dynamic Data Physicalization for Slow Exercising Feedback. In Designing Interactive Systems Conference 2021 (DIS '21), fune 28-fuly 02, 2021, Virtual Event, USA. ACM, New York, NY, USA, 16 pages. https://doi.org/10.1145/3461778.3462041

\section{(c) (i) $\odot$}

This work is licensed under a Creative Commons

Attribution-NonCommercial-NoDerivs International 4.0 License.

DIS '21, fune 28- July 02, 2021, Virtual Event, USA

(c) 2021 Copyright held by the owner/author(s).

ACM ISBN 978-1-4503-8476-6/21/06.

https://doi.org/10.1145/3461778.3462041

\section{INTRODUCTION}

The popularity of running has increased rapidly over the last decades, resulting in it even being one of the most popular recreational sports in the world [49]. Running is characterized by its low threshold and it is attractive for a wide range of people [49]. This heterogeneity among runners is especially represented in terms of age, gender, motivations and abilities [21, 32, 49]. In line with the popularity of running there has been an exponential increase in the development of monitoring devices to support runners in their training [10,21]. Running apps and wearable technologies, such as sports watches, activity trackers and wearable technology, enable quantified data tracking (e.g., pace, distance, route and heart rate) $[9,22,30]$. Based on these data, many of these monitoring devices give feedback through graphs and numbers, aiming at increasing performance during the training $[9,22,56]$ or enabling social and game elements $[3,30,35]$. In these attempts, the design space of how the feedback is communicated tends to be rather homogeneous (i.e., screen based or auditory) [18, 22]. Although this type of feedback offers people a quick glance on their running performance [17] or patterns across time, it does rely on a limited view on the running experience, which already starts before and expands after the running session itself [33]. A recent study suggests that people have high feelings of pride and satisfaction after running, but these positive emotions fade away quickly [33]. An artifact which allows for longer reflection and exploration of running data might support people in experiencing these positive feelings for a longer period [55], going beyond performance-focused feedback.

Recent attempts to communicate activity data tracking in alternative formats have been made, and data physicalizations [20, 64], sometimes using shape-changing interfaces [1, 41], appear as promising. These approaches allow users to explore, share and understand their data differently [20], which in turn encourages reflection on its meaning [64]. In the case of shape-changing interfaces, it changes "from reading and touching flat glass displays to physically manipulating interfaces that transform their shape and materiality to represent the underlying content and context." [1, p.1]. Data physicalizations are not new in design, but implementations of dynamic data physicalizations in real-life cases remain largely unexplored and unexploited due to technical challenges and relative lack of information regarding its value [20]. 


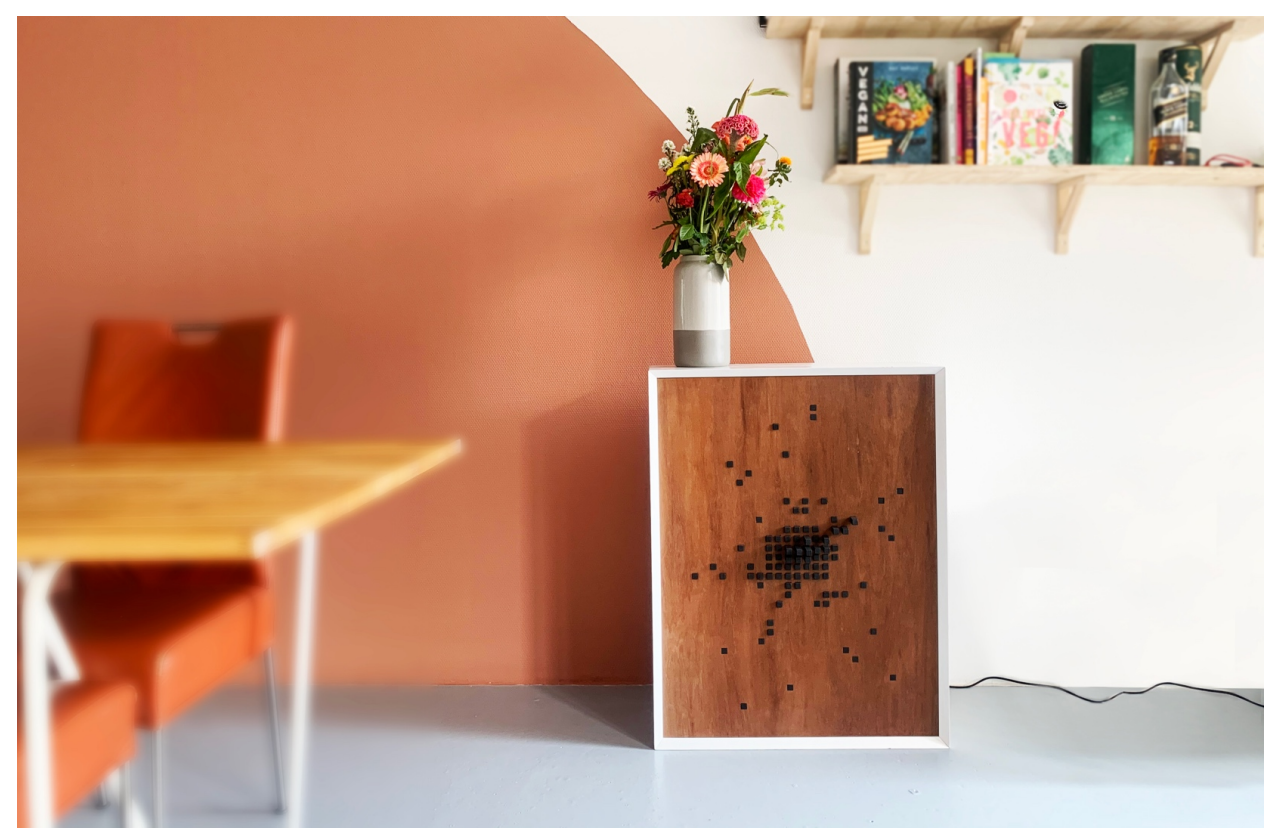

Figure 1: Laina, a shape-changing art piece illustrating previous running routes over a delayed period, placed at participants' home

Following examples of data physicalization in the context of physical activity feedback [24, 25, 31, 55], we designed Laina, a shape changing art piece, presenting physicalized running route data as slow feedback (Figure 1). Through an expert study involving design professionals, we first defined interaction attributes important to slow feedback for running, with a primary focus on reinforcing positive feelings retained from running. We then incorporated these interaction attributes in the design of Laina. Laina explores the promise of data physicalization on outdoor exercise feedback: it allows for a deep reflection, anticipation and exploration of running behavior. Based on the insights collected during the making process and user study, we reflect on the opportunities presented by data physicalization to complement existing running technologies. The main aim of our paper is to inform on the use of a slow feedback mechanism for exercising, through the design of a dynamic data physicalization artefact. Our contributions to the HCI community are the following: 1) defining interaction attributes to materialize a slow feedback mechanism for exercise-related products, 2) exemplifying Laina as a research-through-design artefact embedding those attributes, and 3) providing insights for designing exercise-related products with slow feedback.

\section{RELATED WORK}

\subsection{Data Physicalization on Physical Activity}

Data Physicalization is an emerging research area which main purpose is to convey meaningful information through physical shapes or materiality. It designates " a physical artifact whose geometry or material properties encode data." [20, p.3228]. These physical representations aim at supporting people in exploring, sharing and understanding external or self-quantified data. For Zhao and Vander Moere, this type of information communication goes beyond the data itself, thanks to the tangible nature of the objects, encouraging reflection on its meaning [64]. This is in line with Lockton et al., who stress the need to go beyond quantification as a default mode for information display. They propose the notion of "qualitative interfaces" where "information is presented primarily through representing qualities of phenomena", with the benefit of enabling new forms of understanding [29,31]. Among other opportunities, data physicalizations allow for better use of active perception skills, and thus can easily be explored from different angles [48], but also through different senses (e.g., sound, touch, smell) [20]. Furthermore, opposed to on-screen visualizations, data physicalizations can act as ambient data displays [20]: which are always "on" and placed anywhere. Through their physical presence, data physicalizations also potentially make the data visible to more than oneself (e.g., hidden in a screen or activity tracker), but to others present in that environment, thus stimulating discussions [24].

Data physicalization has been applied in a wide variety of domains [19, 20, 45, 48,57], with a limited number of examples in the context of physical activity feedback $[24,47,55]$. One of these examples is the concept 'Loop' by Sauvé et al. In Loop, physical activity data is presented through an abstract tangible visualization $[46,47]$. Loop consists of changing wooden rings representing the activity goal and the amount of physical activity per days. As an ambient information display, Loop is designed to be incorporated in the physical environment and everyday routine, for users to become more aware of their data [2], or enabling the possibilities of sharing and sociable use through their public availability [62]. Another example of data physicalization in the physical activity domain is TastyBeats [24], a fountain-based system that mixes sport drinks based on the heart rate of the user while exercising.

By presenting data physicalizations in a delayed matter, Activity Sculptures by Stusak et al. [55] proposes a new approach in the 
context of exercise feedback. In this concept, the heartrate of a recreational runner is measured, leading to the generation of an Activity Sculpture, sent to the runner per regular mail two days after the run. This mechanism provided a prolonged rewarding feeling, (i.e. "slow reward mechanism"), reminding the runners of their proud feeling experienced directly after the run. This is an interesting perspective as compared to the continuous stream of data provided by current applications, being just a glance away [17]. Here, slow feedback mechanisms through data physicalizations offer the possibility of anticipation and speculation, which allow for a richer exploration of the data [26, 54].

While being valuable to showcase new ways of using physicality to represent exercising data, these examples entail several limitations. A major limitation for both TastyBeats and Activity Sculptures is the practical feasibility and sustainability concerns. As acknowledged by the designers, producing and sending a physical object to a runner after each session raises issues, which they suggest overcoming by sending one sculpture per month only [55] Similar concerns affect the user acceptance of TastyBeats [24]. Both designs thus act more like an exceptional feedback mechanism than a regular interaction. While most on-screen visualizations are dynamic and interactive, these data physicalization examples thus do not support dynamic data display [20]. Shape-changing interfaces might support the type of feedback offered by data physicalization, while overcoming these limitations. It might offer the possibility to physicalize dynamic data, but also to provide long-term and dynamic user interactions with data $[8,20,53]$.

\subsection{Shape-Changing Interfaces}

Shape-changing interfaces represent a comeback of interaction design in the physical domain [42], envisioning "interactive computational devices to transform into any shape or materiality relevant to the context of use" [1, p.2]. A prime example of this quality in the domain of life sciences is the ability of animals to change shape to scare or hide from enemies [41]. In design research, the adaptive qualities of shape-changing interfaces can be used to enhance the users' interaction [41].

A shape-changing interface can serve different purposes [1]. The main functional purpose of shape changing interfaces is conveying information, meaning or affect by physical change of shape or materiality as in/output $[1,41]$, partly overlapping with the field of Data Physicalization. Since shape-changing interfaces are not restricted to a specific context, examples exist in different applications areas, such as wearable technology [28], (domestic) interactive art-pieces $[37,38,43,63]$, or interactive furniture [6, 7, 31, 38, 51, 60]. A current research focus is also on the technical aspects of shape-changing materials and interfaces [13,39].

In their review of shape-changing interfaces' design space, Rasmussen et al. [41] identified eight types of deformation supporting information and meaning, namely changes in orientation, form, volume, texture, viscosity, spatiality, adding/subtracting, or permeability. These deformations offer a wide variety of interaction possibilities, depending on how the interfaces use physical transformation as an input and/or an output [42]. In an indirect interaction, shape change takes place by implicit input (indirectly controlled by the user), while direct interaction occurs where shape change is part of both the input and output [41]. In the last category, negotiated interaction, the shape change is negotiated between the user and the system [42]. Shape-changing interfaces therefore allow for a degree of openness for change in usage, creating a sense of adaptability to a particular situation and have the potential to enhance interaction with digital information. In their paper, Coelho et al. present four shape-changing design probes, representing different material and deformation properties [8]. The authors envision a series of future application scenarios where shape-changing interfaces act as a tool to enrich human-computer interaction. A key application area identified is the use of form transformation as a representation for dynamic data. shape-changing artefacts where the form and dynamics of interaction are meaningfully intertwined [44]. To understand how interactive devices can engage the body and the mind alike [40], there is a need for robust prototypes - currently scarce - suitable for in-situ evaluations, which would inform on the "suitable contexts of use, the fit between tasks and interfaces, and issues around the cultural appropriation of shape-change" [1, p.5].

In this paper, we further explore how to use a shape-changing interface to represent dynamic running data, while drawing on the work of Stusak et al., by further exploring how to prolong rewarding feelings experienced right after a running session, through a slow feedback mechanism.

\section{DESIGNING A SLOW FEEDBACK MECHANISM}

Designing for slow feedback in a specific context involves a strong consideration for the aesthetics of interaction (e.g. how this type of feedback materializes in an artefact). The Interaction Vocabulary is a dedicated and suitable tool to collect these insights in a structured manner, as aesthetical qualities are typically hard to express to other designers. As a method, the Interaction Vocabulary provides a number of dimensions to describe interaction in design. It is useful for interaction designers as it provides starting points for thinking about the aesthetics of interactions and designing those interactions. The tool, presented as a set of cards, also facilitates communication about intended properties of a product. The Interaction Vocabulary consists of eleven dimensions, each in a pair of opposing attributes. These are descriptive, non-judgmental, non-technology bound attributed of interaction. Each card represents one of the Interaction Vocabulary's dimensions, with the opposite poles on each side of the card. The method can be used in a binary manner, by simply choosing one dimension over the other (e.g. slow rather than fast) or used as a Likert scale to allow for more granularity.

To explore how the concept of a slow feedback mechanism could materialize in a data physicalization artifact, we invited design professionals $(\mathrm{N}=23)$ to participate in a 20 -min online survey study (e.g., asynchronously by themselves). Participants were all interaction and industrial designers familiar with designing for behavior change for an active lifestyle, recruited via professional networks.

Using the Interaction Vocabulary Cards by Diefenbach, Lenz \& Hassenzahl [11], we asked them to describe how a slow feedback mechanism for running would feel/look like with a physical product through an online survey. This method enables designers to think 
Table 1: Attributes used by design professionals $(\mathrm{N}=23)$ to describe a slow feedback mechanism, using the Interaction Vocabulary. To reflect the dichotomic spirit of the original method, the scales were grouped into one or the other dimension, with the opportunity of a neutral option.

\begin{tabular}{|c|c|c|c|c|}
\hline Attribute 1 & $\begin{array}{l}\text { Frequency } \\
\text { Attribute } 1\end{array}$ & Neutral & $\begin{array}{l}\text { Frequency } \\
\text { Attribute } 2\end{array}$ & Attribute 2 \\
\hline Slow & 14 & 2 & 7 & Fast \\
\hline Stepwise & 7 & 2 & 14 & Fluent \\
\hline Instant & 6 & 1 & 16 & Delayed \\
\hline Uniform & 5 & 7 & 11 & Diverging \\
\hline Constant & 11 & 2 & 10 & Inconstant \\
\hline Mediated & 6 & 6 & 11 & Direct \\
\hline Spatial Separation & 2 & 8 & 13 & Spatial Proximity \\
\hline Approximate & 14 & 2 & 7 & Precise \\
\hline Gentle & 9 & 1 & 13 & Powerful \\
\hline Incidental & 0 & 1 & 22 & Targeted \\
\hline Apparent & 14 & 3 & 6 & Covered \\
\hline
\end{tabular}

about the aesthetics of a specific interaction and a way to express it to other designers using a shared vocabulary. To not influence the attributes 'slow' versus 'fast', we provided participants with the following instructions, not explicitly mentioning the notion of 'slow feedback' "Amateur runners indicate to have feelings of high satisfaction and pride right after the end of a session. Unfortunately, they quickly fade over time. How would you design exercising feedback that makes these positive feelings last longer?"

The Interaction Vocabulary consists of 11 semantic differential items to describe the interaction: slow-fast, stepwise-fluent, instantdelayed, uniform-diverging, constant-inconstant, mediated-direct, spatial separation-spatial proximity, approximate-precise, gentlepowerful, incidental-targeted, apparent-covered. The participants were asked to evaluate each pair of words and to indicate which one of the two attributes would better contribute to translate the intended aesthetics of interaction in the design. The participants were asked to evaluate only the pairs of words that they consider relevant to design for a slow feedback mechanism and to leave the others as 'neutral' on the scale. They were also instructed to leave aside aspects that they consider relevant yet dependent on the type of product or use context. After the assessment of the 11 items, we asked the participants to select 3 attributes, out of the aforementioned items, which they consider the most important interaction attributes to design for a slow feedback mechanism, and to explain why, in an open-ended question.

Our results show that eight attributes were associated with a slow feedback mechanism for running in the context of interaction design: slow, fluent, delayed, spatial proximity, approximate, powerful, targeted and apparent (Table 1). Amongst those, delayed (8), powerful (7), slow (5) and targeted (5) were selected as the most important attributes for designing a slow feedback mechanism in the context of running. Other attributes (diverging-uniform, constant-inconstant and direct-mediated) indicated a lower consensus between the attributes, showing high numbers on both sides of the attributes.
The attribute "delayed" was considered important to spark a reminder: "The positive feelings are especially important after the adrenaline kick has faded, when the user is not triggered anymore by the previous workout". This is in line with the attribute "slow": "This would make the satisfaction feeling last longer, because the reward feeling is longer enjoyable." Although most attributes were consistent with the authors' expectations during their initial design explorations, the "powerful" attribute appeared as rather unexpected. One of the designers explains: "The feedback should remind the user that their work-out was powerful". The design experts linked the attribute "targeted" to personalized feedback: "To feel like the feedback is personally made for the user in order for them to emotionally react to it, and to be proud for a longer time."

After the expert study, the authors conducted an interactive and explorative design process, informed by the most important attributes for a slow feedback mechanism elicited through the expert survey (delayed, powerful, slow, and targeted). Besides these attributes, the attribute "approximate" was added during the design process as one of the eight attributes of designing a slow feedback mechanism. Our goal was to design a slow feedback mechanism, that prolongs the rewarding feelings retained from running.

\section{DESIGN CONCEPT: LAINA}

Laina (Figure 1) is a shape-changing art piece, presenting physicalized running routes over a delayed period of one or two days (Figure 2) [55]. Over this time, some of the pins on Laina are pushed out one by one, creating a data physicalization pattern corresponding to the mapping of the last running route. The length the pins come out represent a specific effort given at that point on the route. The metric related to the effort can be chosen by the user (e.g., pace, burned calories, heartrate). Laina adopts an aesthetics perspective to spark positive feelings towards running through a slow feedback mechanism, the art piece revealing itself to the user progressively. Hence, Laina aims to trigger longer satisfaction feelings of the run and reminds the user of the positive emotions of the previous run. 


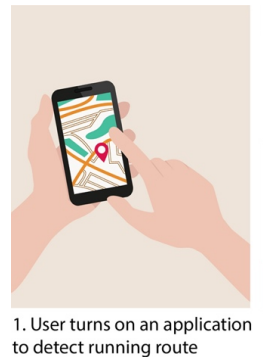
to detect running route

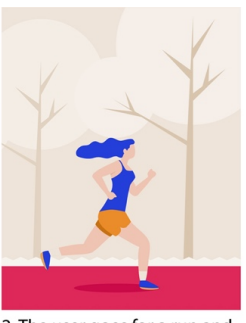

2. The user goes for a run and 3. Laina is activated and starts stops application afterwards to change
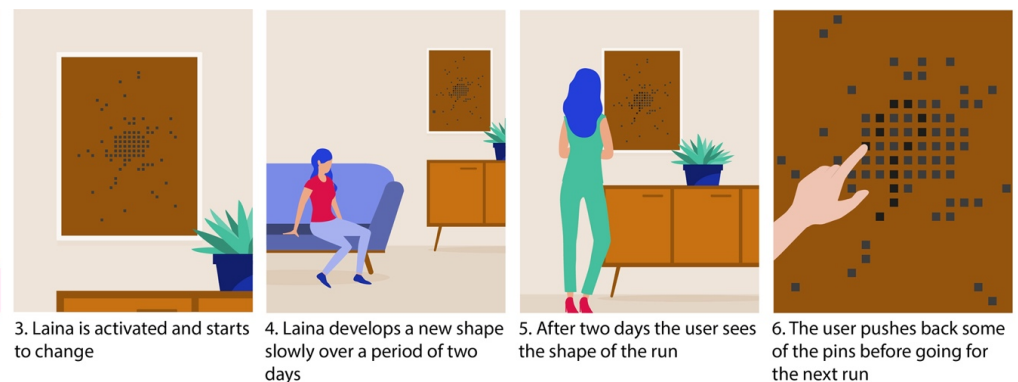

Figure 2: Scenario of use of Laina

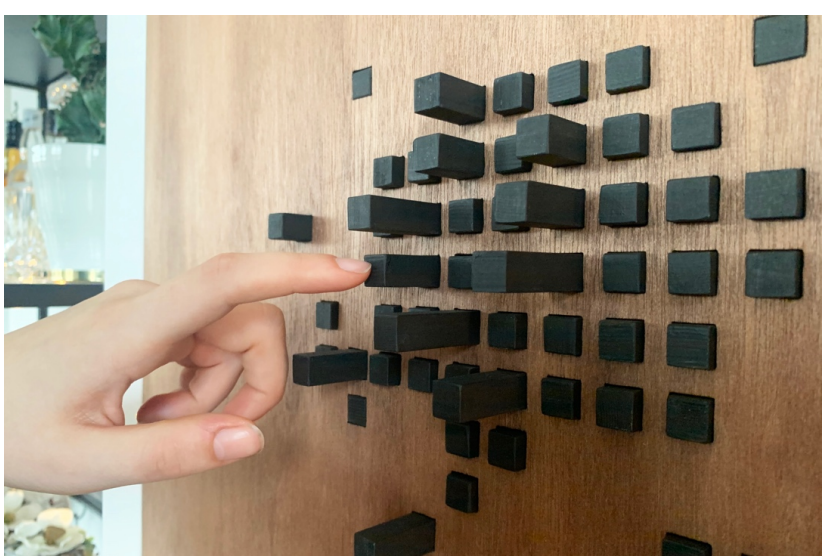

Figure 3: Direct interaction: the user can directly push back the pins as desired

The shape-change interactions of Laina are twofold: indirect and direct interaction. Indirect interaction takes place when the user starts varying their running routes or their level of efforts (e.g., pace, heartrate), the user can indirectly control the shape of Laina, as this affects which of the pins come out and at what length. A direct interaction within Laina was integrated by enabling the possibility of pushing back the individual pins to the original position (Figure 3). If the pins are not pushed back, the different routes will stack on top of each other, showing the joint effort of previous running sessions. Once the maximum depth is reached, the pins do not actuate anymore unless they are pushed back by the user. If some of the pins are pushed back before Laina completes the art piece of the last session, the rest of the running route will still be physicalized in Laina. The direct interaction is integrated to provide autonomy to the user. Hence, the user is in charge of creating their own art-piece, erasing it entirely or keeping certain shapes they already created.

\subsection{Aesthetics of Interaction}

Based on the results of the preliminary survey study, Laina includes five specific Aesthetic of Interaction attributes, which are targeted, delayed, slow, powerful and approximate. We integrated these attributes in Laina in the following manner.

Targeted. Laina uses running route data from external running applications (such as Strava ( ), Runkeeper ( )). Once the session is uploaded, the running route is abstracted (corresponding the "approximate" design choice explained below) and Laina changes shape accordingly, making it a targeted interaction based on tracked data. The pins that will change shape correspond directly to time segments in the user's run. The length of each individual pin is based on the average effort on that time segment

Delayed. Once the user is done with their running session, Laina will not immediately change shape, but will start changing shape after a short delay, when the user does not feel triggered anymore by the previous running session.

Slow. The interaction attribute slow is incorporated in the days Laina needs to fully develop its shape. After one or two days, the user will distinguish the shape of their last running route and might decide to push back some of the pins to change the art-piece.

Powerful. Laina's volume and depth varies according to the length of the pins, which is dependent on the effort given by the user. When a higher level of effort is measured, Laina will turn in a more imposing art piece. Furthermore, as opposed to most activity devices or product, Laina is a relatively big artifact, which further enhances the powerful impression.

Approximate. Both the number and length of pins influence the level of granularity and direct translation of the data physicalized. As pixels in a digital display, more pins would result in more precise data translation. The abstraction of the route through a limited number of pins aligned with our intended aesthetics, relying on an approximate representation supporting discovery and sensemaking.

\subsection{Form and Materials}

Several form and material explorations were made in a researchthrough-design process [65] (Figure 4), leading to the final design of Laina. First, we explored materials that could shape-change as described by Rasmussen et al. [41] (Figure 4) with a focus on investigating possibilities, understanding the constraints of some materials and getting inspiration. We worked for instance with Living Hinge laser cutting material, origami and kirigami techniques or fluidic mechanisms inspired by Venous Materials [34]. We built four intermediate low-fi prototypes (Figure 5), to reflect on different characteristics, namely levels of density, the type of physical change in surface and different forms of restrictions in the visualization of a run. The routes could be physicalized through the density of pins in the black stick model, the color coding of the strings in the metro-map model, or the bendable structure of wood of the Kirigami model or the surfaces in the web of strings model. 

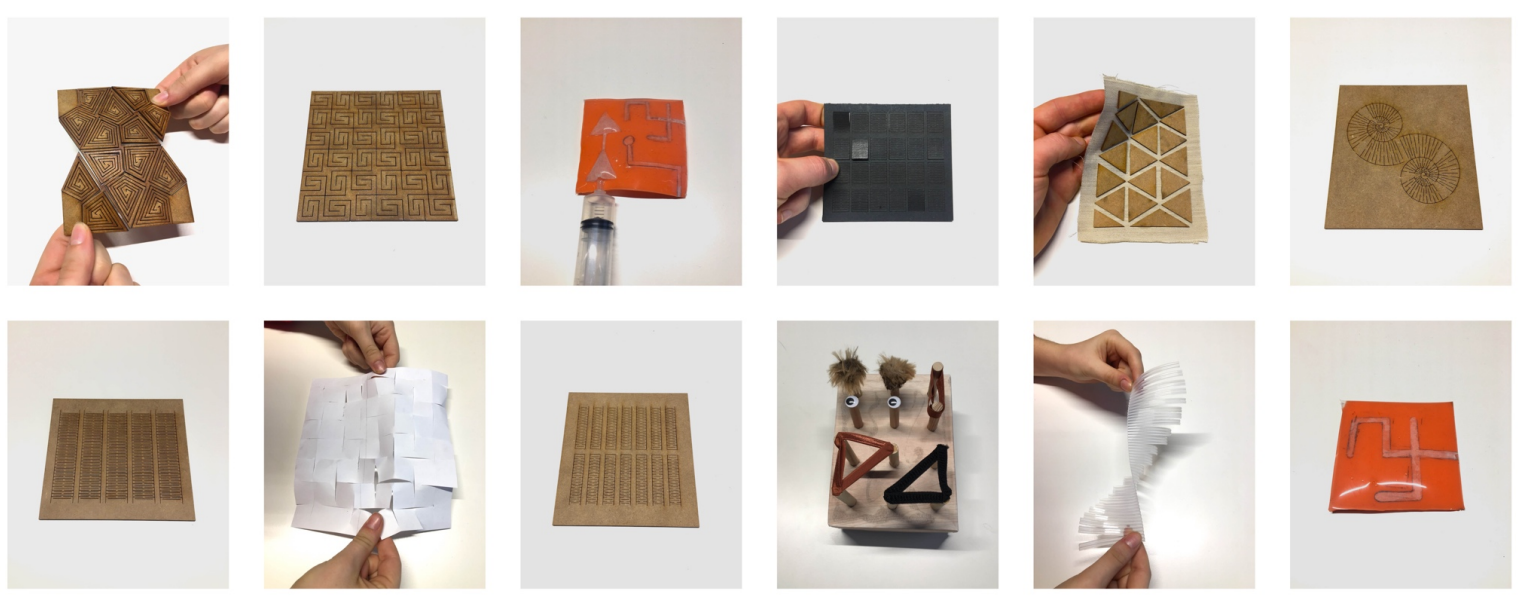

Figure 4: Form and material explorations

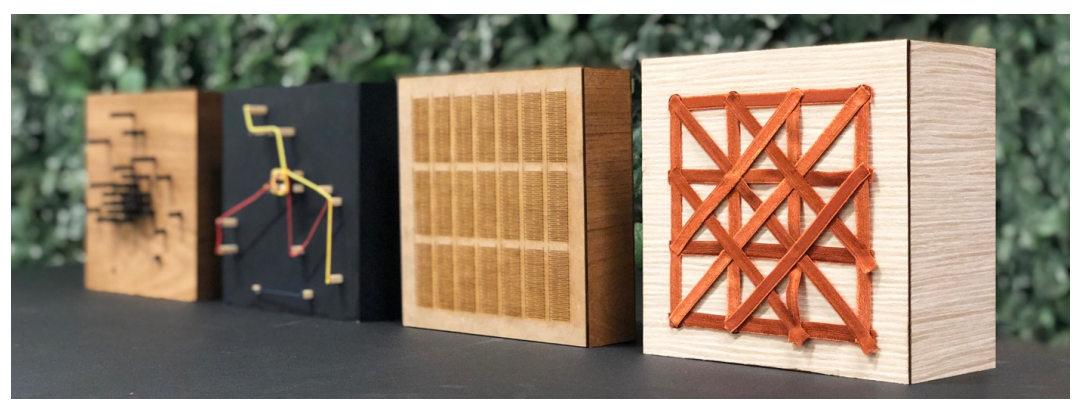

Figure 5: Shape explorations

The level of abstraction and the aesthetical effect created differed greatly according to the prototype, with the Living Hinge being for instance restricted in the visualization supported. Through the different samples, we explored how to incorporate the Aesthetic of Interaction attributes related to a slow feedback mechanism, but also constraints concerning physicalizing running data (Figure 5). We eventually choose the exploration with the wooden black pins, as this system supported certain degree of detail in the visualization while still being able to actuate [61].

In the final design, the visible elements of Laina are composed of three different parts: the case, the front and the pattern (Figure 5). Firstly, the prototype consists of a white case, which is the size of an A1 sheet, and built with wood. We choose this size since this is a widely used size for wall-art. This case is painted with a semi-gloss finish to create a lighter appearance, as well as a contrast to the front. The hard-wood front is the construction with the pattern of the artwork. This is furthermore the part that holds all the pins that change in direction and distort the original shape. The pattern, containing a total of ninety pins, was inspired by a city map of Eindhoven and represents the user in the center of the wall-art. The wooden pins have a black matte finish, each being $10 \mathrm{~cm}$ long. A higher degree of fidelity and aesthetics was achieved mainly for the user to be able to contextualize the concept and design, but also to increase people's acceptance of Laina in their everyday domestic environment [62].

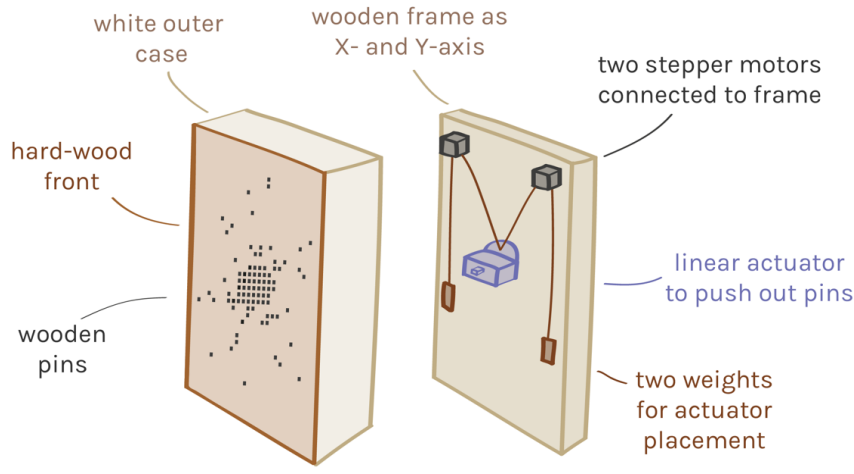

Figure 6: Overall architecture of hardware of Laina

\subsection{Technology and Realization}

Overall Architecture. The overall realization architecture of Laina consists of a wooden frame, supported by a wooden plate at the back, which serves as a base for an X-and Y-axis (Figure 6). Two stepper motors are placed on top of this wooden frame, controlling the position of a linear actuator (Figure 7, middle).

Hardware. The linear actuator is responsible for pushing the pins out, making it possible to control each individual pin (Figure 7, right). The same pin can be pushed out multiple times, showing 


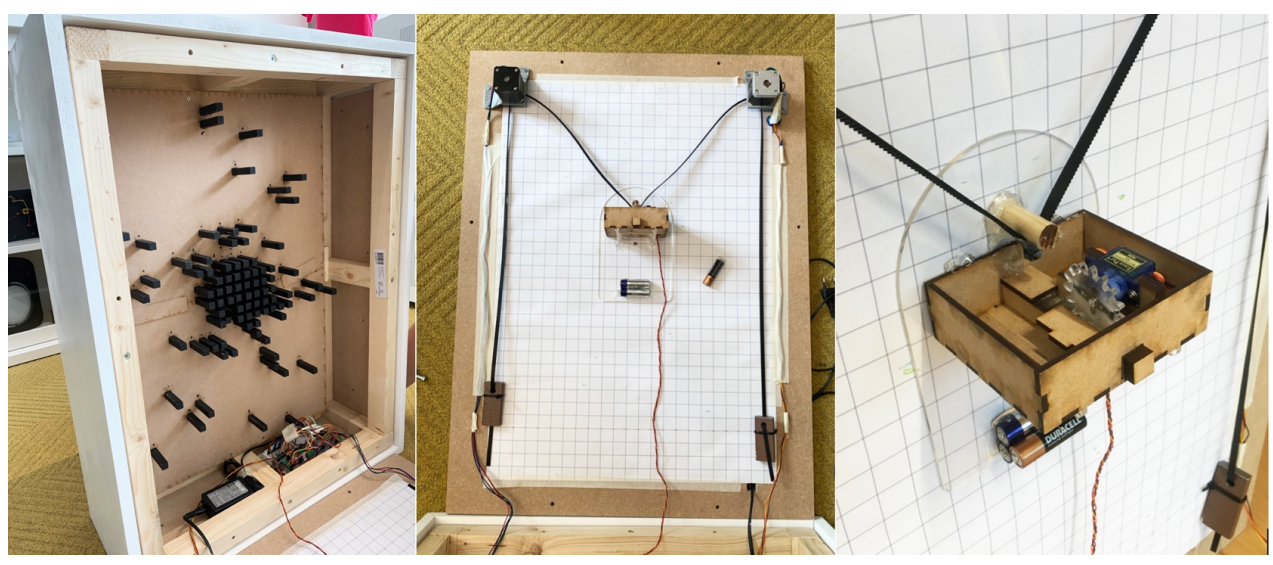

Figure 7: Left: inside of front of Laina. Middle: two stepper motors placed at the two top corners of the frame. Right: Linear actuator responsible for moving the individual pins

incremental feedback, until the maximum depth of shape-change is reached $(4.5 \mathrm{~cm})$. To make the pins go back to its original state, it should be pushed back by the user as part of the direct interaction. The position of the linear actuator on the $\mathrm{X}$ - and Y-axis is controlled by two stepper motors, which are placed on the frame, allowing the linear actuator to move to each desired individual pin (Figure 7).

Software. To control the linear actuator and its position, we used the microcontroller ESP32-WROOM which communicates over WiFi. The microcontroller gets all the data from a real-time Firebase database which gets updated by a running node script and webpage. In the current prototype, the transformation from the detected route (via an external platform like Strava $\odot$ or Runkeeper $\odot$ ) to the physicalization of the route on Laina is not automated. To solve this, a webpage was created where the pins could be altered by the researchers (Figure 8). The researchers hence mediated remotely once the running session was completed and abstracted the route, choosing pins directly corresponding to time segments in the user's run. The length of each individual pin is based on the average effort on that time segment (e.g., averaging pace, heartrate) and can be controlled on the webpage. By clicking on the selected pin on the webpage, the researcher indicates the 'push factor' (e.g., how much the pin should be pushed, ranging from level 1 to 5). The push level is based on an average of the effort during the run. The average pace of the run is used as a baseline and correspond to a push factor of 3. One level above or below, will indicate an increased or decreased effort of $10 \%$. The threshold of $10 \%$ per level is based on the variability of running pace in our population of recreational runners. With 2 levels above or below the average pace, the system can acknowledge efforts of $+/-20 \%$ in average pace. When all the pins are selected and the push factor is entered in the system, the research clicks on 'Execute' at the bottom of the page, to send the abstracted route to the Firebase database connected to Laina. The database includes an algorithm that calculates the timing of pushing out each individual pin, and only operates between 9:00 until 21:00, with a maximum total of two days to push out the entire route (depending on the time the running session has ended). Where in the art piece the abstract route is presented, depends on the place the user runs: a long, far run will for instance be visualized further away from the center in the art piece. The mapping ratio is relative to the user's previous sessions. Eventually in future work, the transformation from the route should be fully automated to make the product use sustainable.

\section{METHOD: USER STUDY}

The intention of this study was not primarily to assess the concept of Laina, but the aim was to use Laina as a research artefact to gain insights on how a slow feedback mechanism using exercise data physicalization, was experienced. More specifically, how this would prolong rewarding feelings right after a running session, through a slow feedback mechanism. We deployed Laina in 3 households for a 3-week field study with each participant. We combined several pre- and post-interviews conducted at the participants' houses with a diary study. In line with other qualitative research in the design field concerning in-depth, in-situ observations in the domestic environment [14, 16, 27], this formed the core of our methodology. The first field study was conducted in Winter 2020. The additional two user studies were conducted in Summer 2020 (note: the participants were home more often due to Covid-19). This study and its design have been approved by the ethical board of the university, and informed consent was obtained among the participants.

\subsection{Data Collection and Analysis}

The study consisted out of three parts: (i) an introduction interview, (ii) logging daily experiences and (iii) a debriefing interview of Laina. All interviews and diary logs were transcribed verbatim, then coded and analyzed with MaxQDA Analytics Pro 2018 by thematic analysis using an inductive approach. The first author subsequently created an initial coding scheme, after which consensus was sought among the researchers to derive a final coding scheme. Quotes used in this paper were translated from Dutch to English. Each interview took about 100 minutes and was audio recorded. After explaining the objectives of the study, the participants did sign an informed consent form for their participation. Participants were not instructed about minimum time to exercise or interact with 


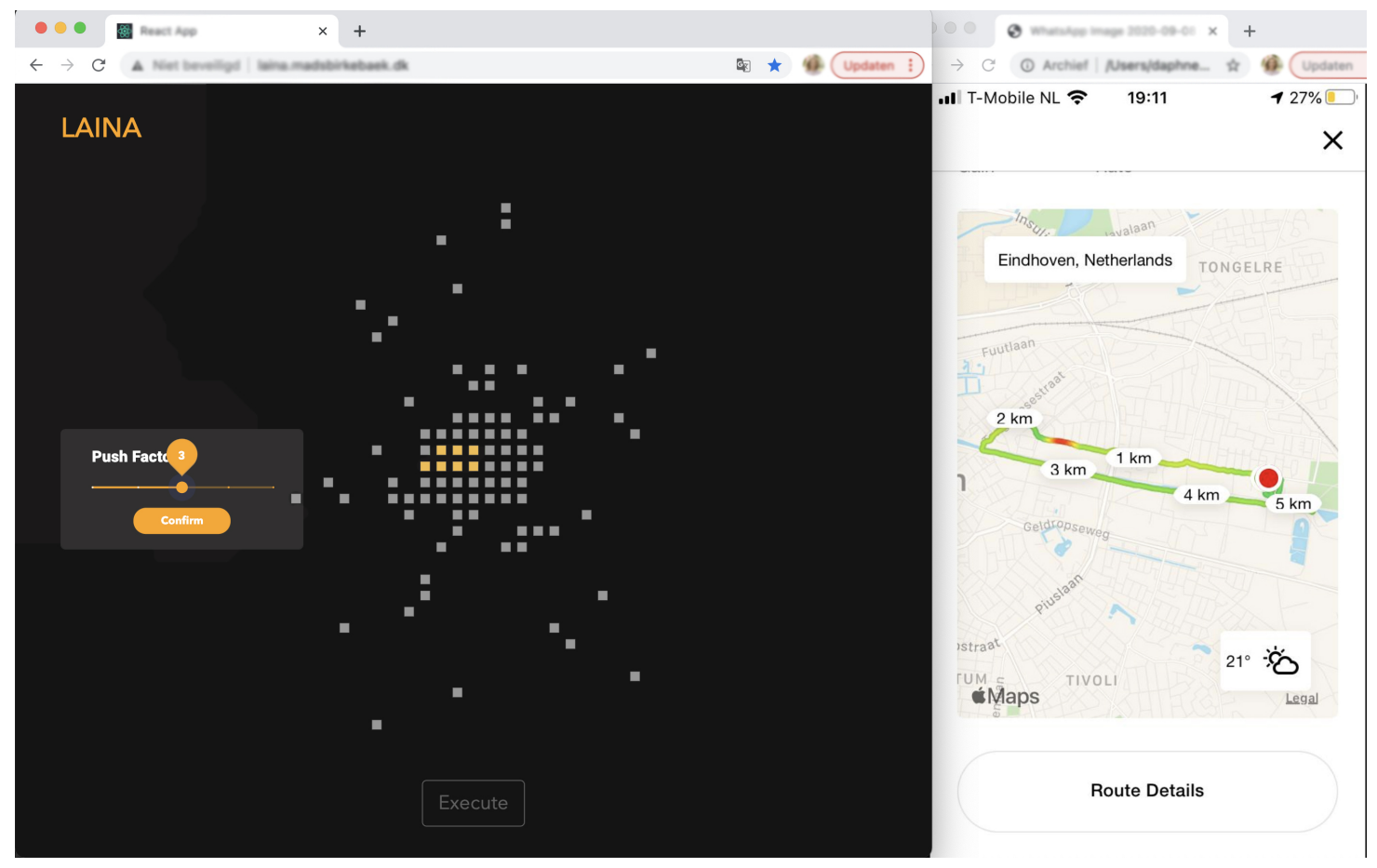

Figure 8: Webpage to control Laina's shape- changing interface. Each individual pin can be selected while indicating the 'Push Factor' (affecting the length of the pin, corresponding to the chosen effort). The orange dots correspond to the selected pins, that will be pushed out. Once the abstracted route is indicated, the 'Execute' button sends the data to Laina

the device. No additional details about the meanings were given (besides the fact that Laina represents a route and pins can be pushed to "reset" it). It was left open to them to discover what the length of the pins represented. In the following sections we describe the procedure of each type of data collection.

5.1.1 Introduction Interview. In the introduction interview, we invited the participant to describe their running behavior (e.g., goals, frequency of training, experience) and the devices currently used to support trainings. We aimed at better understanding the lifestyles, running routines (e.g. "how long have you been running?"; "how often did you run last week?", "what is important for you when you run?"), motivational factors ("do your intentions often match your actions?" "are there any objects in your house that motivates you to go running?") and the role of technology in a running session. After this interview, we asked the participant what interior means to them and where they would like to place the object. We installed Laina at a place of own choice in the living room (Figure $1 \& 9$ ). We then explained the role of the object in the following way "Laina will represent the route of a run. The route is extracted from your running platform account." At this point, we engaged into a discussion first asking what their first impression of the product was. Then, we used the Product Reaction Cards [4], which are part of the Desirability toolkit by Benedek and Miner. Microsoft's product reaction cards are a customizable list of 118 words used to evaluate any product or concept. It is particularly useful for physical product evaluation after a field study or user test. Using Product Reaction cards eases the evaluation process from the users' viewpoint, while providing a comparison basis between participants. Product Reaction Cards can be printed out and used during a face-to-face session. They can also be included in an online survey. People are asked to choose words from the list, that would in their opinion describe the product (Figure 10). This constitutes an ideal base for discussion in an interview setting [4]. We used the reduced list of 64 words by Neil Turner [59]. Finally, the discussion revolved around the expectations for the coming testing period. At the very end, we explained that it is possible to erase the pattern on Laina by pushing the sticks back when feeling like it.

5.1.2 Logging of daily experiences. To report on daily experiences with Laina and associated feelings, the participants were asked to log their observations, by sharing a voice message on the instant messaging application WhatsApp and a picture of the current shape of Laina. Voice messaging was used to allow more freedom of expression and ensure that the participants would not feel restricted by a written diary and could express thoughts smoothly, as they flew.

5.1.3 Debriefing interview. After the in-situ deployment period, we conducted a semi-structured debriefing interview. We enquired about how the participants experienced Laina across time, how they interpreted this experience and how it potentially influenced their behavior and attitude. After a few questions, we showed the participant the data that was logged during the diary in order to help recalling memories and experiences, and help us make sense of their meaning-making processes. 
Table 2: Participants Overview

\begin{tabular}{llllll}
\hline Participant & Age & Household & Running Experience & Running Technology & $\begin{array}{l}\text { Frequency per } \\
\text { week }\end{array}$ \\
\hline P1 & 45 & Married, with two children & 8 years & Garmin + Strava & $3-4$ times \\
P2 & 27 & Single, living with mother & 2 months & Strava & $2-3$ times \\
P3 & 30 & Relationship, living together & 15 years & Nike Running Club & 3 times \\
\hline
\end{tabular}

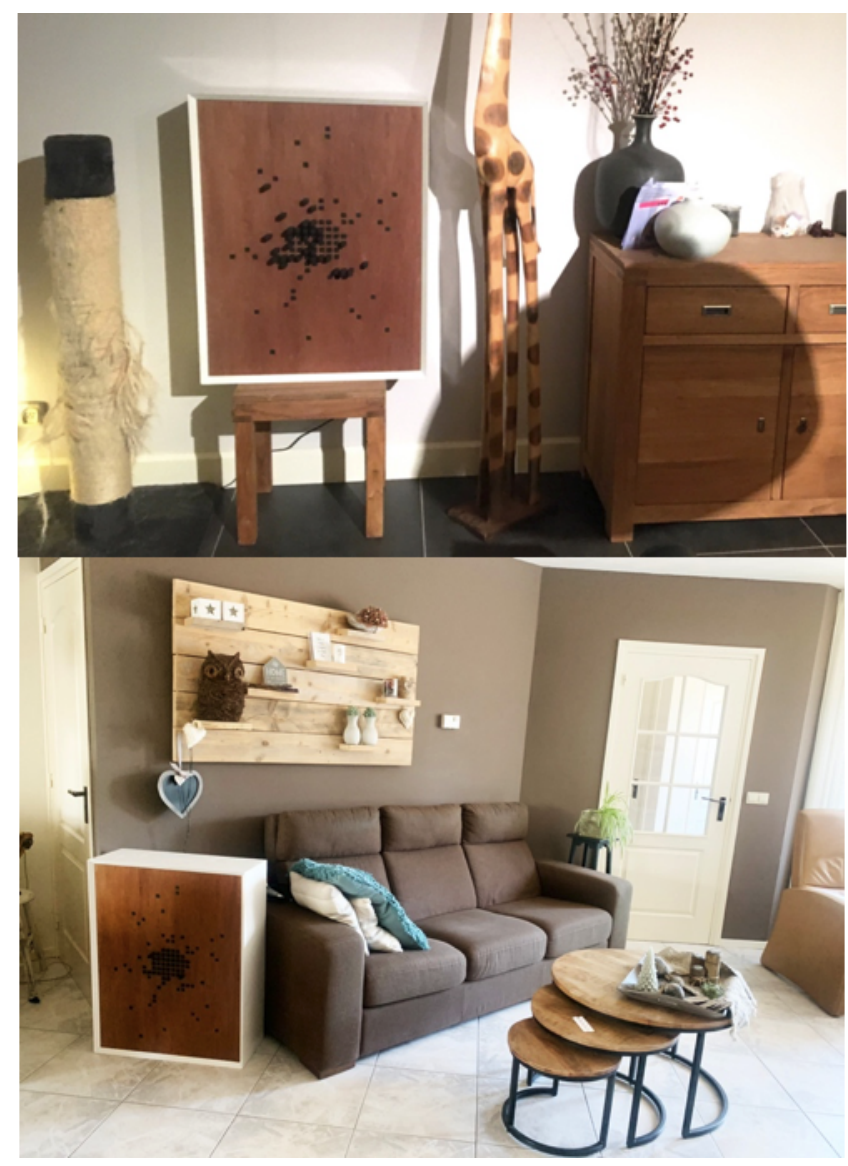

Figure 9: Top, Laina at P1, placed on a small table. Bottom, Laina placed in P2's living room.

\subsection{Participants}

The field study was conducted with three participants, recruited via a local running group page or personal pages of the researchers on social network. The participants expressed an interest in participating in the study and were selected purposively because of the insights they could bring to the topic. The participants were rewarded with a $€ 30$ voucher for their participation. All participants identified as women but were diverse in age, household composition and running experience (Table 2). All participants work for at least 32 hours per week and sit a lot during work. They all had in common to not value the performance and competition elements of running. This varied from valuing the activity of running itself instead over being faster than herself or others (P1), clearing one's

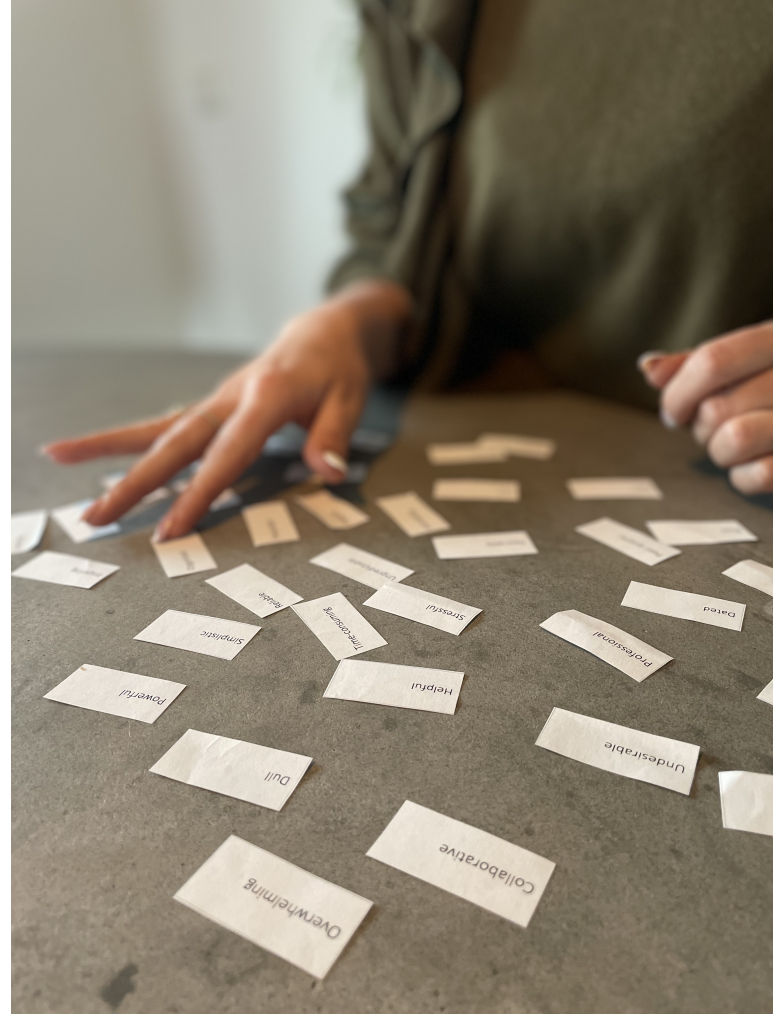

Figure 10: Product Reaction Cards in use

head (P3) or just being active (P2). While running, all participants tracked their run, automatically uploading their sessions to a social running platform. Although participants looked at these results briefly after the running sessions, they all admitted to not looking at it very often.

\section{RESULTS}

\subsection{Introduction Interview}

All participants first talked about themselves. Afterwards they talked about running routines, motivational factors, the role of running technology and their first impression of Laina.

6.1.1 Running Routines, Technology and Motivational Factors. All participants expressed an aim to run three times a week, with P1 running about four times a week, in addition to fitness: "So if I do all my training it is 6 times a week, but sometimes it's "okay, not today!". P2 just started running after the gyms closed due to 
Covid19 and is still in search of her running routine: "I wanted to keep exercising, and this was the only thing I could do". Only P3 indicated that running was her primary sport: "Sometimes I change the time or day when I eventually go running, but I do make sure I run at least three times a week". As P2 just started running, she tried to not focus on time and speed, but rather on the total distance. P3 explains that she really runs to clear her head, "I know that often, going for a run, is exactly what I need at that moment, because I know I will feel better afterwards". Despite several injuries which made P1 stop running at several times in the last 8 years, she stayed motivated and adapted her pace. She is training for a running event which is her main motivation, but "I don't know if I am going to manage" she states. Regarding the use of running technology, P1 uses a smartwatch linked to a running platform but admits that she "does not look very often". Contrary to P1, P2 indicated to value the social elements of the platform: "I value Strava because other people can see it. This is stupid because you actually have to run for yourself, but I do like the fact that other people can see it." P3 also uses a running application: "During running, I'm busy thinking a lot about things that are happening now. So sometimes I do these Guided Runs by Nike, to be occupied with that during running."

6.1.2 First Impression. P1 decided to place Laina on a small table (Figure 9), P2 and P3 decided to put it on the ground. After being briefly explained what to expect from Laina, P1 immediately expressed curiosity: "I' $m$ very curious what's going to come out, what's going to happen if I have done a run". When being asked to choose some cards from the Product Reaction Cards [4] to describe their first impression, the words chosen at least twice were powerful, simplistic, innovative, inviting and attractive. Powerful: "It really has a presence, if it was smaller you would be able to look past it or forget about it" - P2. "I think it looks really cool with the black and the pins, and I feel it has a sense of power to it." - P3. Simplistic: "I really think it looks minimalistic, with the white and black, so it looks rather simple" - P2. Innovative \& Inviting: "These ones catch my eye first. By all means, I think it is "innovative", I have never seen it before.... I understand there will be different patterns, or a pattern as a whole. Which also means it is "inviting", to do what needs to be done to activate it." - P1. Attractive: "I like the simplistic look of it, and I feel that it is very attractive. It looks cool and minimalistic" P3. Words that were chosen only once were entertainment, not a common thing, overwhelmed, difficult, comfortable, professional, unpredictable and intimidating. Words as overwhelmed, difficult and unpredictable were chosen because of the unexpectedness of what Laina would do: "Maybe because I don't have a clue on what it will do or what I have to do with it." - P3.

When talking about the black pins, both $\mathrm{P} 1$ and $\mathrm{P} 3$ recognized the map of the city in the visualization: "What I already thought is, it looks a little bit like [anonymized for review]. I thought maybe it is just the city center and kind of the districts around." - P1. When realizing it represents the city map, all the participants started brainstorming on how the runs will potentially affect Laina. P2 explains: "Well from here, because I really live in the city center, so I would take a lap to the south, or one going north. And then when I'll do my 7.5k run I would reach these 2 pins, because I would run like this (pointing to the map on Laina). However, I wouldn't reach the west side, because I don't really run there often." - P2

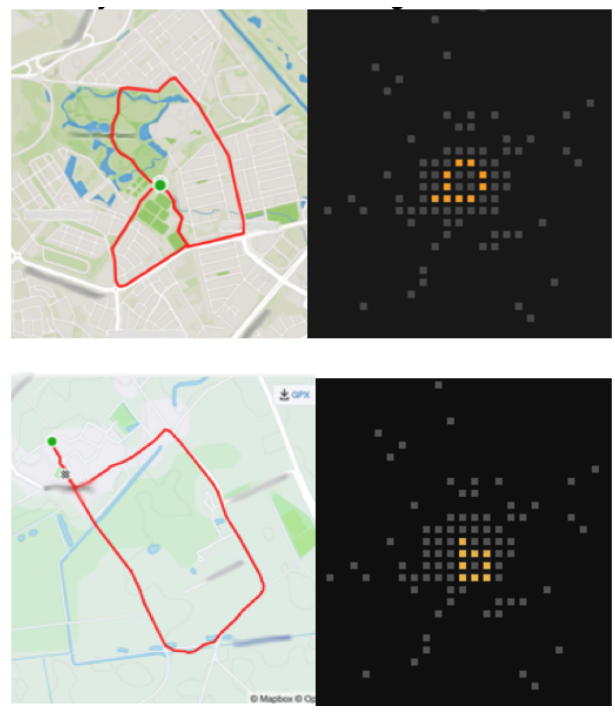

Figure 11: Two visualizations of two first running routes on running platform and their abstracted input for Laina in the black. On the top P1 and on the bottom P2.

\subsection{Logging Daily Experiences}

In our protocol, we used the logging of daily experiences in the debriefing interview to help recalling memories and experiences. We also retrieved insights from the participants' voice memos.

Recognizing Route. On the first morning after installing Laina, all participants went for a run, curious to experiment how it would work. After the first shape change, P1 started to see a circle in the physicalization of Laina. However, when comparing this to her visualized route on the running platform she felt that it was not one-to-one aligned and thought that her route was not looking like a circle. This confused her: "I thought it (referring to physicalization on Laina) was going to be a circle, but it (referring to running route on Strava) is not a circle at all." (Figure 11). This was different for P2 and P3 who recognized their running routes in the physicalization yet expected it to appear faster: "I think I see my running route, although I expected to see it immediately when I came back from running but that happened over different phases. Every time it was doing something it was nice and I immediately checked how it changed"P3.

Length of Pins. Another element that was quickly noticed by the participants was the different lengths of the pins. Although participants choose their most important effort, they were not informed the length of the pins would represent this. P2 did recognize the differences in her effort on the route: "I think the length of the pins represents my speed. Because at a certain point I could see I was faster, and when I think of my route I know I was faster there because that was a spot where I saw other people and I wasn't feeling like seeing other people so then I always run faster." After some days, P1 also started to notice the difference in pins lengths and wonders if all eventually will come out. The length would then reflect the speed or distance: "It seems that some of them are coming out further, so I'm wondering is it coming out because of your speed (then it's not so very fast, so it was a slow run Saturday), or is it because of 
Table 3: Themes emerging during the debriefing interview analysis

\begin{tabular}{lll}
\hline Theme & Subtheme & Sub-subtheme \\
\hline Data Physicalization and Shape Changes & Indirect Interaction & $\begin{array}{c}\text { Anticipation } \\
\text { Comparison } \\
\text { Recognition }\end{array}$ \\
& & Direct Interaction \\
& Physical Data \\
Slow Feedback Mechanism & Reminding \\
& Timing \\
& Reward & Reflection \\
Look \& Feel & Sound & Furniture \& Placement \\
\hline
\end{tabular}
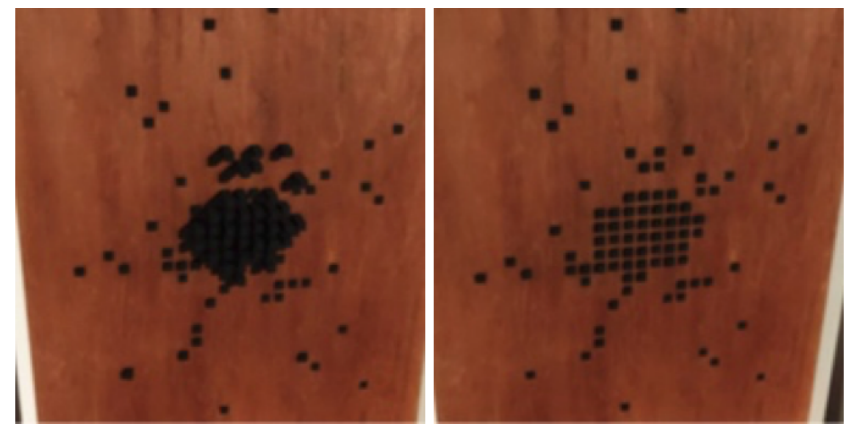

Figure 12: Pictures of P1: Before (left) and after (right) pushing back all the pins right before a long-distance run

your distance (well, not further then $10 \mathrm{~km}$, so it's also very centralized)." P3 also started wondering about it: "I'm not sure yet what the length of the individual pins means, how far they are pushed out. I was thinking maybe it represents my speed but I'm not certain about it."

Resetting Laina. After almost 2 weeks, P1 and P3 decided to push back all the pins right before going for a next run (Figure 12), enabling to better see how this would affect the visualization. They were satisfied with the outcome: "It's really nice to get feedback again, the last few days I didn't really notice a lot of differences so it's really nice to be able to recognize my running route clearly again." - P3. Only P2 had a clear vision of wanting to push back the pins every week: "Because I don't run that far and often yet, I don't want to push them back every time I go for a run. But I agreed with myself to push back the pins every week, I don't know why."

\subsection{Debriefing Interview}

In the debriefing interview, we asked participants how they experienced Laina. Based on our interview sessions, our approach was to construct themes that captures important perceptions and experiences with Laina and their running behavior. For each single interview, we coded pieces of texts (e.g., quotes) which relate to a part of the phenomenon and clustered these in different themes. The three emerging themes with subthemes are presented in Table 3. The focus of the analysis was on understanding the perception of the physicalizations and level of shape change control, as well as how the slow feedback mechanism was perceived and eventually impacted the experience.

\subsubsection{Data Physicalization and Shape Changes.}

Indirect Interaction. All participants indicated they started running in different routes than normally, challenging Laina to change shapes to different sides, or even outside the center "I consciously took different routes than I normally do. Normally I always run the same rounds" - P1. Although they all identified their running routes in Laina's physicalization, they were uncertain what the length of the pins represented exactly. However, in their daily loggings they all linked it to effort on specific points of the run (e.g., speed or heartrate). P2 mentioned to remember this by watching Laina: "Without Laina I would not have noticed it, but it is because I have been observing the feedback much closer, that I realized that I run faster when I see other people".

At some point all participants indicated that it was hard to recognize their individual runs, as the runs were stacked on top of each other, especially when parts of different running routes overlapped. The lack of new physical feedback (in forms of new pins) even sparked feelings of guilt in P3, despite having a regular running routine, and having the pins pushed out further: "Because I didn't really receive new visual feedback, I have the feeling I didn't do anything for a long time".

However, the fact of being able to indirectly control the shape change of Laina also triggered anticipation right before going for a new run: "Right before I go, I'm wondering what I can do to make a new pattern and I'm really anticipating how the eventual feedback will look like." - P3. P2 even considered of going for another run the same day, to also include a specific pin that is in reach: "I was thinking, well I could go for another round to try and push that pin out too". This was also the case for P1, who had a strong urge to push out pins beyond the center of Laina, triggering anticipation: "I just wanted the pins outside the center to also come out."

Direct Interaction. P1 and P3 did not determine beforehand when they would push back the pins of Laina, while P2 immediately decided to make it a weekly effort: "I really try to run three times a week, and I'm not sure if I'm able to really run more than three different routes in one month, so that would mean I have a lot of double routes". The other participants first wanted to wait and see 
what happens, while also appreciating that it showed the mutual effort of the different runs, which increased feelings of pride on their accomplishment: "At the end I thought like: wow look at all those pins that came out, look at what I have accomplished" - P3. Similar to P2, P1 and P3 eventually systematically stacked effort weekly: "What I would do next, I think, is let the running routes stack on top of each other. Then I would be able to see how my week looked like. Or specifically with a special running session" - P1.

All participants felt they had a 'clean slate' after pushing back all the pins. P1 explains: "By pushing it in you start with a clean slate again". Both P1 and P3 explained to regret not doing that earlier, because it made it easier to compare how the shape exactly changed: "I should have done it before, so I would have been able to more easily see the different patterns". Although, the individual routes became less visible, the tradeoff might have been a desire to push out more pins by two of the participants.

By pushing back the pins, two participants explained that this raised their curiosity again: "Because you start from a clean slate again, you regain curiosity again ... I was wondering what would happen this time." - P1. This direct control was valued: "I valued the possibility of pushing the pins ... there is a certain freedom in choosing to remove a part, or everything. But there are more variables this way". This was also appreciated by P3, who indicated she would probably not remove pins that took more effort: "I would not push the pins back that are all the way at the top, I would probably keep those."

Physical Data. The fact that Laina gave physical feedback was considered as one of the most appreciated elements by all the participants. "You can also actually touch something (talking about pushing back the pins), so you feel more connected to it." - P1. The presence in their domestic environment increased feelings of pride: "I'm proud because I'm doing well, and Laina made that very visible, because it is not digital" - P3. This physical feedback also removed a concrete understanding of the actual performance but added a sense of ambiguity to the feedback: "Because you don't see your concrete pace or distance. Normally I'm quite critical towards myself, thinking I was slow for example. And with this (Laina), you don't feel like that but you see that you did something, and that is enough." - P3. This was the same for P2, who experienced that she actually changed her negative feelings to positive feelings: "The run didn't go well and I was really done with it but then there was still a lot happening with this (Laina). And although it wasn't my best performance, I realized it wasn't that bad because I did go running, and I actually went exercising today, so instead of being bummed all day, I felt good about it". Although Laina represented physical running data, all participants indicated to consider Laina as a part of their furniture: "At some point it was just considered as one of our pieces of furniture." $-\mathrm{P} 1$

6.3.2 Slow Feedback Mechanism. Initially, all participants expected the feedback to be fast and instant: "I expected it would come immediately after running but the feedback came in phases" - P3. This was confusing for P1, who ran 11 times during the 3 weeks field study. She thus was not aware of the intentional slow feedback given, since many pins overlapped in time: "I really noticed that my previous run wasn't visualized fully and then suddenly the next already arrived". She therefore felt Laina was too slow in giving feedback:
"I think it would have been nicer if the feedback was presented faster. If you go for a run, that it for example would start an hour after and then it should be finished soon.". She did however indicate this had to do with her intensive running schedule. She did feel the feedback was complementary to the running technologies she uses: "After every training I analyze it with Garmin. But this Saturday I was like; 'Nice an additional pattern on Laina".

The feedback was experienced differently by P2 and P3, who valued the slow and delayed timing. Because the feedback would come in 'phases', they were more often reminded of their previous effort and it felt rewarding: "Because it was presented in different phases, you are reminded of your achievements more often, and that makes me proud. Because in a sense, I pushed out those pins. I could also not go, and then nothing would have happened." - P3. For P2 this was the aspect she valued the most about Laina: "I really like that it comes during the day. It would be a pity if it would come at once. ... Then you would be done with it immediately. While I really noticed that I was reminded of my run, and sometimes I just went and checked whether something has happened. If it comes at once, you glance at it and that's it. You then won't further explore it. At least I wouldn't." - P2. This delayed feedback also allowed for a deeper reflection on their previous running route: "And then I was thinking like: Oh yes that was that part on the route! And this was here. And then you start to think about your running session again." - P3, and a prolonged rewarding feeling "I look back at Laina, and then I think, yes that was fun and a good achievement, it acts as a reminder" - P3.

6.3.3 Sound. Laina in its current form is using stepping motors to change the position of the linear actuator to move an individual pin. The motors sound caused some frustration to P1 and also her family members: "During dinner it made quite some noise." "It was quite annoying when we were watching TV." Contrary to this, both P2 and P3 expressed they felt happy and reminded of the previous run every time they heard Laina making a sound, and explained it functioned more as a notification that a new physical feedback arrived: "The fact that it reminds you, because you went for a run, and I don't really like to go for a run, but afterwards you always feel great. And every time I heard a little sound, the feeling I normally get afterwards from running, came back to me. ... and this combination with the sound triggered me to go look at the prototype more often." P2.

Because of the sound, Laina triggered social interactions within the household of P1. This happened when she was not home herself, but her partner was: "When I came home, I was always curious, especially when my partner notified me that during the day the shape had changed".

\section{DISCUSSION}

In this paper, we explored the experience of a shape-changing interface as slow exercise feedback in the domain of recreational running. We intended to unveil dynamic data physicalizations and their potential to support novel human-computer interactions, where the aesthetics of interaction supports new ways of understanding and making sense of personal data. We thus extend the design space of exercise-related products and services, which currently follows on the market a rather standardized approach in form and feedback. To learn about how data is felt and interpreted beyond a 
screen-based device giving quantified feedback, we designed Laina, a shape-changing art piece relying on data physicalization and acting as a slow feedback mechanism.

Designing for a slow feedback mechanism in the context of running is a challenging endeavor, which we addressed in two ways. First, by involving design professionals $(\mathrm{N}=23)$ we reflected on how the concept of a slow feedback mechanism in the context of running could materialize in a design artifact, using the Interaction Vocabulary [11]. Second, by embedding and materializing these interaction attributes in the design of an interactive art piece, we gained insights into the effect of dynamic data physicalizations and slow feedback mechanisms on exercising.

Thanks to our robust prototype Laina, we have been able to deploy medium term in-situ user tests. We thus undertook one of the challenges of evaluating shape-changing interfaces, which devices are often too "fragile, hard to replicate, and not suitably robust for long term use" [1, p.5]. As mentioned by Alexander et al., in-situ evaluations of shape-change in real-life are needed and will help to understand the match between tasks and interfaces, as well as the essential dimension of appropriation of shape-changing artifacts [1]. Our user study hence enabled us to assess the suitability of a shape-changing interface as slow exercise feedback through an in-depth examination of the participants experience and brought a holistic view and rich insights on these experiences.

\subsection{Reflection-in-action and Reflection-on-action}

Laina did both stimulate reflection-in-action and reflection-onaction, encouraging anticipation and exploration. In our study, anticipation took place when the participants were preparing to go for the next run, but also when they examined the art-piece and tried to run new routes to create new shapes. Regarding reflection-in-action [50], they also indicated to think about affecting the art-piece during a running session. Although recreational runners often reflect on what route they will take beforehand, using Laina conferred a different intention to this thought (i.e. to influence the shape). The participants even mentioned being "challenged" by the art piece. Reflection-on-action consists of reflecting retrospectively, after a running session, on how the run affected the physical visualization. However, the results show that Laina also offered a sense of ambiguity in the feedback, raising curiosity to explore new routes, and anticipation of their impact. The benefit of incorporating ambiguity in exercise feedback is illustrated in previous work $[5,12,36]$ : it leaves people a necessary space to interpret situations for themselves and "encourages them to start grappling conceptually with systems and their contexts, and thus to establish deeper and more personal relations with the meanings offered by those systems." [15].

\subsection{Physical Feedback}

Laina was placed at a prominent spot in the participants' living room during the user tests. As opposed to running applications, tangible artifacts can be incorporated in the physical environment and users' daily routines [2, 14, 16, 62]. The participants indicated that Laina was quickly considered as a piece of furniture. While they admitted to rarely look at their running data on their tracking apps, Laina's physical presence triggered more moments to remind oneself of previous runs. This especially happened when a running session was different from the previous ones (if still visible). The smooth incorporation into people's physical environment is expected to compensate for the natural decrease in novelty and stimulation feelings as documented in user experience theories [23]. Thanks to its tangible presence in the living room, Laina can become a permanent reminder, that will not trigger vivid feelings of curiosity but will become a companion reminding of one's exercising endeavor.

Being able to physically engage with Laina (touching the feedback), participants felt more connected to their data. They also noted that a physical interface showing their approximate run removed a sense of judgement on how the running session went, but emphasized being responsible for pushing out the pins, prolonging a sense of pride. "Because in a sense, I pushed out those pins. I could also not go, and then nothing would have happened." P3. Participants valued the ability to physically stack individual runs too, showing a cumulative effort, to reset when desired or keeping specific pins representing an effort. In some cases, the physical presence of pins thus triggers how one felt, at a specific time during a run, highlighting a new perspective to data reflection. As opposed to digital interfaces, this bodily engagement thus encouraged connectedness, pride and reflection.

\subsection{Indirect Interaction}

Since the shape-changes in Laina were not one-to-one representations of the actual route, the visualization was perceived as more implicit and ambiguous. This raised mixed emotions among the participants, and P1 expressed feelings of confusion, yet accompanied by curiosity. A solution could be to increase the number of pins that physicalize the running route for a more targeted translation of the data. However, this ambiguity was also valued by some participants, as it facilitated a shift from the actual performance to the fact of just "going for a run". The ambiguity also triggered participants to get the pins on the outer side of the interface to be pushed out, and to challenged Laina in different ways: first by intensifying efforts, then by increasing the distance and finally by running in different zones in the city. This indirect interaction was designed intentionally to understand its effects and to initiate a dialog between the artifact and the user: the participants had to explore multiple running routes and level of efforts to get an idea on how this might dynamically affect the physicalization. This is typically not supported by static data physicalization artifacts, which communicate the information in a unidirectional way. Shape-changing interfaces there have the key advantage of being able to acquire new forms, which in themselves can carry new meanings [8]. This form of indirect interaction is a challenging endeavor since: "Inferring user's actions and intentions is generally not a straightforward matter." [42, p.206], but enables a sense of magic to it allowing both the user and the product to transform. We therefore intended running sessions to be a negotiation between what the user wants and what Laina conveys.

\subsection{Direct Interaction}

Rasmussen et al. state that the transparency of the interaction and the control offered to the user is often neglected when deploying 
shape-changing interfaces [42]. Although the user is offered control over the shape change, it still might be unclear to the user what can and will happen if they interact with the object. This was also mentioned by our participants during the debriefing interviews, expressing regrets about not having directly interacted with Laina earlier. We choose to balance usability and discovery, by providing some basic information to the user while still keeping a sense of openness and exploration opportunities. The temporality of the interaction plays an important role, as the user learns and adapts to the product over time. This was also the case for Laina, where over three weeks, the participants started to expect some specific shapes to appear when going for a specific running route and indicated to have learned from the interaction.

\subsection{Complementary to other Feedback Mechanisms}

Through our field studies, we realized that Laina acted complementary to current feedback schemes. Indeed, the status-quo on exercising feedback is rather instant after the training or even inthe-moment [17]. This enables people to reflect upon their exercise activity immediately after, or during the session. Laina offers the possibility of a longer reflection timespan. Since the feedback is presented slowly, our participants reflected on how the routes were represented in the art piece but were also reminded of their accomplishments with pride. The power of this reminding element is in line with the concept of Activity Sculptures, where delayed feedback was considered as an additional reward mechanism [55] Shape-changing interfaces better support these forms of feedback mechanisms. Thanks to their dynamism, they allow to overcome the sustainability and feasibility challenges posed by static data physicalizations [8]. Combined with existing exercise-related technologies, shape-changing artifacts have the potential to support exercise feedback. Overall, interventions using complementary touchpoints might be more effective to address the challenges of behavior change for physical activity.

\subsection{Limitations}

Our study entails several limitations. In Laina, we deformed running route data to translate it into a data physicalization artefact. The physicalization on Laina however might still be considered as a 'dequantified conversion' of the visualization presented on the running application. The use of qualitative interfaces, where a phenomenon is represented as closely related to the actual behavior [29], might open up even more opportunities for people to explore and reflect based on the level of 'directness of connection' proposed by Lockton et al. [29].

While our prototype was robust enough for an in-situ deployment, one can admit that the material used for Laina does not entail complex mechanical properties. Laina only relies on an "orientation" deformation type and does not suffer risks related to reversibility (i.e. the capacity of the material to fully recover from the shape memory transitions without considerable decay). The field of soft mechanics, referring to shape-changing materials and their composites, has many more challenges to overcome to generate physical transformation via transition through different memory and elasticity states [8]. New developments in this domain would thus bring even more exciting opportunities for interaction design and data physicalization.

In our study, we choose to investigate the experience of the interaction with Laina through an in-depth user study involving three participants during a 3-week deployment period. While this could be seen as a limitation in some disciplines, small sample sizes is not uncommon in design studies [27, 58], with a focus on in-depth examination of personal experience and meaning-making activities case-by-case [52]. In a domain where human-computer interactions are largely unexplored, we therefore do not aim to overgeneralize our results. Besides learning through making, by taking a researchthrough-design approach [65] we aimed at presenting a detailed examination of user experiences and at providing in-depth insights rather than focusing on the incidence of a specific observation. Future studies using complementary approaches to the investigation of users' experiences are of course needed. It is through the triangulation of perspectives that the community will grow and realize the promise of maximizing the benefits of data physicalization and shape-changing interfaces in promising application domain such as health, public spaces, robotic and many more.

\section{CONCLUSION}

In this paper, we explored how dynamic data physicalizations can open up the design space of outdoor exercise feedback and inform how to design for a slow feedback mechanism in the running context. Following examples of data physicalization in the context of physical activity, we designed Laina, a shape-changing art piece acting as a slow feedback mechanism for recreational runners. We deployed Laina at 3 participant's home, during a series of 3-weeks field studies, exploring how data physicalizations might support exercise feedback. In our study this dynamic feedback allowed for longer reflection, anticipation and exploration of running behavior. Based on the insights collected during the making process and user study, we discussed the opportunities presented by data physicalizations to complement existing running technologies. In this paper, we intended to unveil the promise of dynamic data physicalizations and their potential to support novel human-computer interactions, where the aesthetics of interaction supports new ways of understanding and making sense of personal data. Both the design and study of Laina contribute to extending the design space of outdoor exercise-related products and services.

\section{ACKNOWLEDGMENTS}

We would like to thank all the participants who contributed to this study. Additionally, we also thank Nicolai Hansen for his worthwhile comments on the paper. This work is part of the project Nano4Sports, which is financed by Interreg Vlaanderen-Nederland.

\section{REFERENCES}

[1] Jason Alexander, Anne Roudaut, Jürgen Steimle, Kasper Hornbæk, Miguel Bruns Alonso, Sean Follmer, and Timothy Merritt. 2018. Grand challenges in Shapechanging interface research. Conference on Human Factors in Computing Systems - Proceedings 2018-April: 1-14. https://doi.org/10.1145/3173574.3173873

[2] Saskia Bakker, Elise van den Hoven, and Berry Eggen. 2015. Peripheral interaction: characteristics and considerations. Personal and Ubiquitous Computing 19, 1: 239254. https://doi.org/10.1007/s00779-014-0775-2

[3] Christine Bauer and Simone Kriglstein. 2015. Analysis of Motivation Strategies in Running Tracking Applications. Proceedings of the 13th International Conference 
on Advances in Mobile Computing and Multimedia - MoMM 2015: 73-79. https: //doi.org/10.1145/2837126.2839316

[4] Joey Benedek and Trish Miner. 2002. Measuring Desirability: New Methods for Evaluating Desirability in a Usability Lab Setting. Proc. UPA 2002: 5.

[5] Boudewijn Boon, Janjaap Van Der Net, Marco Rozendaal, Pieter Jan Stappers, and Marry M. Van Den Heuvel-Eibrink. 2016. Playscapes: A design perspective on young children's physical play. In Proceedings of IDC 2016 - The 15th International Conference on Interaction Design and Children, 181-189. https://doi.org/10.1145/ 2930674.2930713

[6] Arielle Chapin. 2017. Interactive wall: Dynamic structure in living spaces. TEI 2017 - Proceedings of the 11th International Conference on Tangible, Embedded, and Embodied Interaction: 739-743. https://doi.org/10.1145/3024969.3035532

[7] Marcelo Coelho and Pattie Maes. 2009. Shutters: A Permeable Surface for Environmental Control and Communication Marcelo. In Proceedings of the Third International Conference on Tangible and Embedded Interaction (TEI'09), Feb 16-18 2009, Cambridge, UK Shutters: https://doi.org/10.1093/nq/s10-IX.213.66-d

[8] Marcelo Coelho and Jamie Zigelbaum. 2011. Shape-changing interfaces. Personal and Ubiquitous Computing 15, 2: 161-173. https://doi.org/10.1007/s00779-0100311-y

[9] Ashley Colley, Pawel W. Wozniak, Francisco Kiss, and Jonna Häkkilä. 2018. Shoe integrated displays: A prototype sports shoe display and design space. ACM International Conference Proceeding Series: 39-46. https://doi.org/10.1145/3240167. 3240216

[10] Joan Martine Dallinga, Matthijs Mennes, Laurence Alpay, Harmen Bijwaard, and Marije Baart De La Faille-Deutekom. 2015. App use, physical activity and healthy lifestyle: A cross sectional study. BMC Public Health 15, 1: 1-9. https: //doi.org/10.1186/s12889-015-2165-8

[11] Sarah Diefenbach, Eva Lenz, and Marc Hassenzahl. 2013. An Interaction Vocabulary. Describing the How Of Interaction. Conference on Human Factors in Computing Systems - Proceedings 2013-April: 607-612. https://doi.org/10.1145/ 2468356.2468463

[12] Elisabeth Kersten van Dijk, Joyce Westerink, and Wijnand IJsselsteijn. 2016. Deceptive Visualizations and User Bias: a Case for Personalization and Ambiguity in PI Visualizations. The 2016 ACM International foint Conference on Pervasive and Ubiquitous Computing: Adjunct: 588-593. https://doi.org/10.1145/2968219.2968326

[13] Sean Follmer, Daniel Leithinger, Alex Olwal, Akimitsu Hogge, and Hiroshi Ishii. 2013. InFORM: Dynamic Physical Affordances and Constraints through shape and object actuation. UIST 2013 - Proceedings of the 26th Annual ACM Symposium on User Interface Software and Technology: 417-426. https://doi.org/10.1145/2501988 2502032

[14] William Gaver, John Bowers, Andy Boucher, Andy Law, Sarah Pennington, and Nicholas Villar. 2006. The history tablecloth: Illuminating domestic activity. Proceedings of the Conference on Designing Interactive Systems: Processes, Practices, Methods, and Techniques, DIS 2006: 199-208.

[15] William W Gaver. 2003. Ambiguity as a Resource for Design. In In Proceedings of the SIGCHI conference on Human factors in computing systems, 233-240.

[16] William W. Gaver, Albrecht Schmidt, John Bowers, Anthony Steed, Andrew Boucher, Nicholas Villars, Hans Gellerson, Brendan Walker, and Sarah Pennington. 2004. The Drift Table: Designing for ludic engagement. In Confer ence on Human Factors in Computing Systems - Proceedings, 885-900. https: //doi.org/10.1145/985921.985947

[17] Rúben Gouveia, Fábio Pereira, Evangelos Karapanos, Sean A. Munson, and Marc Hassenzahl. 2016. Exploring the design space of glanceable feedback for physical activity trackers. Proceedings of the 2016 ACM International foint Conference on Pervasive and Ubiquitous Computing - UbiComp '16: 144-155. https://doi.org/10. 1145/2971648.2971754

[18] Bas Van Hooren, Jos Goudsmit, Juan Restrepo, and Steven Vos. 2019. Realtime feedback by wearables in running: Current approaches, challenges and suggestions for improvements. Fournal of Sports Sciences (RFSP) 00, 00: 1-17. https://doi.org/10.1080/02640414.2019.1690960

[19] Steven Houben, Connie Golsteijn, Sarah Gallacher, Rose Johnson, Saskia Bakker, Nicolai Marquardt, Licia Capra, and Yvonne Rogers. 2016. Physikit: Data engagement through physical ambient visualizations in the home. Conference on Human Factors in Computing Systems - Proceedings: 1608-1619. https://doi.org/10.1145/ 2858036.2858059

[20] Yvonne Jansen, Pierre Dragicevic, Petra Isenberg, Jason Alexander, Abhijit Karnik, Johan Kildal, Sriram Subramanian, and Kasper Hornbæk. 2015. Opportunities and Challenges for Data Physicalization. In CHI '15 Proceedings of the 33rd Annual ACM Conference on Human Factors in Computing Systems, 3227-3236.

[21] Mark Janssen, Jeroen Scheerder, Erik Thibaut, Aarnout Brombacher, and Steven Vos. 2017. Who uses running apps and sports watches? Determinants and consumer profiles of event runners' usage of running-related smartphone applications and sports watches. PLoS ONE 12, 7: 1-17. https://doi.org/10.1371/journal. pone. 0181167

[22] Mads Møller Jensen and Florian Floyd Mueller. 2014. Running with technology: Where are we heading? In Proceedings of the 26th Australian Computer-Human Interaction Conference on Designing Futures: the Future of Design, 527-530.
[23] Evangelos Karapanos, John Zimmerman, Jodi Forlizzi, and Jean Bernard Martens. 2009. User experience over time: An initial framework. In Conference on Human Factors in Computing Systems - Proceedings, 729-738. https://doi.org/10.1145/ 1518701.1518814

[24] Rohit Ashok Khot, Jeewon Lee, Deepti Aggarwal, Larissa Hjorth, and Florian Floyd Mueller. 2015. Tasty beats: Designing palatable representations of physical activity. Conference on Human Factors in Computing Systems - Proceedings 2015April: 2933-2942. https://doi.org/10.1145/2702123.2702197

[25] Rohit Ashok Khot, Ryan Pennings, and Florian Floyd Mueller. 2015. EdiPulse: Supporting physical activity with chocolate printed messages. Conference on Human Factors in Computing Systems - Proceedings 18: 1391-1396. https://doi. org $/ 10.1145 / 2702613.2732761$

[26] Rohit Ashok Khot, Simon Stusak, and Andreas Butz. 2017. 10 design themes for creating 3D printed physical representations of physical activity data. In IFIP Conference on Human-Computer Interaction, 85-105.

[27] Matthias Laschke, Marc Hassenzahl, Jan Brechmann, Eva Lenz, and Marion Digel. 2013. Overcoming procrastination with ReMind. In In Proceedings of the 6th International Conference on Designing Pleasurable Products and Interfaces, 77. https://doi.org/10.1145/2513506.2513515

[28] Young Suk Lee. 2015. Spiky Starfish: Exploring "felt technology" through a shape changing wearable bag. TEI 2015 - Proceedings of the 9th International Conference on Tangible, Embedded, and Embodied Interaction: 419-420. https://doi.org/10. 1145/2677199.2690878

[29] Dan Lockton, Delanie Ricketts, Shruti Aditya Chowdhury, and Chang Hee Lee. 2017. Exploring Qualitative Displays and Interfaces. In CHI Conference Extended Abstracts on Human Factors in Computing Systems, 1844-1852. https://doi.org/10. $1145 / 3027063.3053165$

[30] Matthew Mauriello, Michael Gubbels, and Jon E Froehlich. 2014. Social Fabric Fitness: The Design and Evaluation of Wearable E-Textile Displays to Support Group Running. In Proceedings of the SIGCHI Conference on Human Factors in Computing Systems, 2833-2842. https://doi.org/10.1145/2556288.2557299

[31] Daphne Menheere, Ida Damen, Carine Lallemand, and Steven Vos. 2020. Ivy: A Qualitative Interface to Reduce Sedentary Behavior in the Office Context. In In Proceedings of DIS '20 Companion, July 6-10, 2020, Eindhoven, Netherlands.

[32] Daphne Menheere, Mark Janssen, Mathias Funk, Erik Van Der Spek, Carine Lallemand, and Steven Vos. 2020. Runner's Perceptions of Reasons to Quit Running: Influence of Gender, Age and Running-Related Characteristics. International Journal of Environmental Research and Public Health 17, 6046: 12.

[33] Daphne Menheere, Carine Lallemand, Erik van der Spek, Carl Megens, Andrew Vande Moere, Mathias Funk, and Steven Vos. 2020. The Runner's Journey: Identifying Design Opportunities for Running Motivation Technology. In Proceedings of NordiCHI'20.

[34] Hila Mor, Tianyu Yu, Ken Nakagaki, Benjamin Harvey Miller, Yichen Jia, and Hiroshi Ishii. 2020. Venous Materials: Towards Interactive Fluidic Mechanisms. Conference on Human Factors in Computing Systems - Proceedings: 1-14. https: //doi.org/10.1145/3313831.3376129

[35] Florian Floyd Mueller, Chek Tien Tan, Rich Byrne, and Matt Jones. 2017. 13 Game Lenses for Designing Diverse Interactive Jogging Systems. In Proceedings of the Annual Symposium on Computer-Human Interaction in Play, 43-56.

[36] Florian Floyd Mueller, Frank Vetere, Martin R. Gibbs, Darren Edge, Stefan Agamanolis, Jennifer G. Sheridan, and Jeffrey Heer. 2012. Balancing exertion experiences. In Conference on Human Factors in Computing Systems - Proceedings, 1853-1862. https://doi.org/10.1145/2207676.2208322

[37] Sara Nabil, Aluna Everitt, Miriam Sturdee, Jason Alexander, Simon Bowen, Peter Wright, and David Kirk. 2018. ActuEating: Designing, studying and exploring actuating decorative artefacts. DIS 2018 - Proceedings of the 2018 Designing Interactive Systems Conference: 327-340. https://doi.org/10.1145/3196709.3196761

[38] Sara Nabil and Richard MacLeod. 2020. Peace: Projecting dual-identities on interactive furniture. TEI 2020 - Proceedings of the 14th International Conference on Tangible, Embedded, and Embodied Interaction: 837-848. https://doi.org/10. $1145 / 3374920.3375006$

[39] Ken Nakagaki, Luke Vink, Jared Counts, Daniel Windham, Daniel Leithinger, Sean Follmer, and Hiroshi Ishii. 2016. Materiable: Rendering dynamic material properties in response to direct physical touch with shape changing interfaces. Conference on Human Factors in Computing Systems - Proceedings: 2764-2772. https://doi.org/10.1145/2858036.2858104

[40] Marianne Graves Petersen, Ole S Iversen, and Peter Gall Krogh. Aesthetic Interaction - A Pragmatist 's Aesthetics of Interactive Systems. Learning.

[41] Majken K. Rasmussen, Esben W. Pedersen, Marianne G. Petersen, and Kasper Hornbæk. 2012. Shape-changing interfaces: A review of the design space and open research questions. In Proceedings of the SIGCHI Conference on Human Factors in Computing Systems: 735-744. https://doi.org/10.1145/2207676.2207781

[42] Majken Kirkegård Rasmussen, Timothy Merritt, Miguel Bruns Alonso, and Marianne Graves Petersen. 2016. Balancing user and system control in shapechanging interfaces: A designerly exploration. TEI 2016 - Proceedings of the 10th Anniversary Conference on Tangible Embedded and Embodied Interaction: 202-210. https://doi.org/10.1145/2839462.2839499 
[43] Yvonne Rogers, William R. Hazlewood, Paul Marshall, Nick Dalton, and Susanna Hertrich. 2010. Ambient Influence: Can Twinkly Lights Lure and Abstract Representations Trigger Behavioral Change? In UbiComp'10, September 26-29, 2010, Copenhagen, Denmark, 261-270. https://doi.org/10.1145/1864349.1864372

[44] Philip R. Ross and Stephan A.G. Wensveen. 2010. Designing behavior in interaction: Using aesthetic experience as a mechanism for design. International fournal of Design 4, 2: 3-13.

[45] Kim Sauvé, Saskia Bakker, and Steven Houben. 2020. Econundrum: Visualizing the Climate Impact of Dietary Choice through a Shared Data Sculpture. 12871300 .

[46] Kim Sauvé, Saskia Bakker, Nicolai Marquardt, and Steven Houben. 2020. LOOP: Exploring Physicalization of Activity Tracking Data. In Proceedings of the 11th Nordic Conference on Human-Computer Interaction: Shaping Experiences, Shaping Society, 1-12. https://doi.org/10.1145/3419249.3420109

[47] Kim Sauvé, Steven Houben, Nicolai Marquardt, Saskia Bakker, Bart Hengeveld, Sarah Gallacher, and Yvonne Rogers. 2017. LOOP: A physical artifact to facilitate seamless interaction with personal data in everyday life. DIS 2017 Companion Proceedings of the 2017 ACM Conference on Designing Interactive Systems: 285-288. https://doi.org/10.1145/3064857.3079175

[48] Kim Sauvé, Dominic Potts, Jason Alexander, and Steven Houben. 2020. A Change of Perspective: How User Orientation Influences the Perception of Physicalizations. 1-12. https://doi.org/10.1145/3313831.3376312

[49] Jeroen Scheerder, Koen Breedveld, and Julie Borgers. 2015. Running across Europe the rise and size of one of the largest sport markets. Springer.

[50] Donald A. Schon. 1983. The Reflective Practitioner: How Professionals Think in Action. Basic Books: N. Y.

[51] Mark Selby and David Kirk. 2015. Experiential Manufacturing: The Earthquake Shelf. Research Through Design, March: 25-27. https://doi.org/10.6084/m9.figshare. 1327994.Experiential

[52] Jonathan A. Smith. 2017. Interpretative phenomenological analysis: Getting at lived experience. Fournal of Positive Psychology 12, 3: 303-304. https://doi.org/10. 1080/17439760.2016.1262622

[53] Paul Strohmeier, Antonio Gomes, Giovanni Maria Troiano, Aske Mottelson, Timothy Merritt, and Jason Alexander. 2016. Sharing perspectives on the design of shape-changing interfaces. Conference on Human Factors in Computing Systems - Proceedings 07-12-May-: 3492-3499. https://doi.org/10.1145/2851581.2856478

[54] Simon Stusak. 2016. Exploring the Potential of Physical Visualizations.

[55] Simon Stusak, Aurelien Tabard, Franziska Sauka, Rohit Ashok Khot, and Andreas Butz. 2014. Activity sculptures: Exploring the impact of physical visualizations on running activity. IEEE Transactions on Visualization and Computer Graphics 20, 12: 2201-2210. https://doi.org/10.1109/TVCG.2014.2352953

[56] Jakob Tholander and Stina Nylander. 2015. Snot, Sweat, Pain, Mud, and Snow: Performance and Experience in the Use of Sports Watches. Proceedings of the ACM CHI'15 Conference on Human Factors in Computing Systems 1: 2913-2922. https://doi.org/10.1145/2702123.2702482

[57] Alice Thudt, Uta Hinrichs, Samuel Huron, Sheelagh Carpendale, Télécom Paristech, and Université Paris-saclay. 2018. Self-Reflection and Personal Physicalization Construction. Proceedings of the SIGCHI Conference on Human Factors in Computing Systems - CHI'18: 1-13. https://doi.org/10.1145/3173574.3173728

[58] Lucy Tindall. 2009. J.A. Smith, P. Flower and M. Larkin (2009), Interpretative Phenomenological Analysis: Theory, Method and Research . . Qualitative Research in Psychology 6, 4: 346-347. https://doi.org/10.1080/14780880903340091

[59] Neil Turner. Product Reaction Cards Reduced List. Retrieved April 22, 2021 from http://www.uxforthemasses.com/product-reaction-cards/

[60] Luke Vink, Viirj Kan, Ken Nakagaki, Daniel Leithinger, Sean Follmer, Philipp Schoessler, Amit Zoran, and Hiroshi Ishii. 2015. TRANSFORM as Adaptive and Dynamic Furniture. 183-183. https://doi.org/10.1145/2702613.2732494

[61] Hannes Waldschütz and Eva Hornecker. 2020. The Importance of Data Curation for Data Physicalization. In In Proceedings of DIS '20 Companion, Fuly 6-10, 2020, Eindhoven, Netherlands, 293-297. https://doi.org/10.1145/3393914.3395892

[62] Salu Ylirisku, Siân Lindley, Giulio Jacucci, Richard Banks, Craig Stewart, Abigai Sellen, Richard Harper, and Tim Regan. 2013. Designing web-connected physical artefacts for the 'aesthetic'of the home. Conference on Human Factors in Computing Systems - Proceedings: 909-918. https://doi.org/10.1145/2470654.2466117

[63] Bin Yu, Nienke Bongers, Alissa van Asseldonk, Jun Hu, Mathias Funk, and Loe Feijs. 2016. LivingSurface: Biofeedback through Shape-changing Display. In TEI 2016 - Proceedings of the 10th Anniversary Conference on Tangible Embedded and Embodied Interaction, 168-175. https://doi.org/10.1145/2839462.2839469

[64] Jack Zhao and Andrew Vande Moere. 2008. Embodiment in data sculpture: A model of the physical visualization of information. Proceedings - 3rd International Conference on Digital Interactive Media in Entertainment and Arts, DIMEA 2008: 343-350. https://doi.org/10.1145/1413634.1413696

[65] John Zimmerman, Jodi Forlizzi, and Shelley Evenson. 2007. Research through design as a method for interaction design research in HCI. Conference on Human Factors in Computing Systems - Proceedings: 493-502. https://doi.org/10.1145/ 1240624.1240704 